\title{
Condrosarcoma cervical alto
}

\author{
C.J. Domínguez; S. Martín-Ferrer; J. Rimbau y C. Joly
}

Servicio de Neurocirugía. Hospital Universitario Dr. Josep Trueta. Gerona.

\section{Resumen}

Los condrosarcomas espinales son tumores extremadamente raros y de crecimiento lento. Su principal localización es toraco-lumbar y cervical baja ${ }^{10}$. El caso que nosotros presentamos, una mujer, tiene un tumor localizado en la zona cervical alta $\mathrm{C} 2$ con extensión caudal a C3 y a lo largo de su evolución hasta C4 de predominio derecho. A pesar de haberse publicado con anterioridad ${ }^{1}$ aportamos nuevos aspectos de estabilidad cervical instrumentada y comparamos el resultado obtenido con las series publicadas.

PALABRAS CLAVE: Condrosarcoma. Tumor espinal. Columna cervical. Inestabilidad.

\section{Upper cervical chondrosarcoma}

\section{Summary}

Spinal chodrosarcoma is extremely rare and slowly growing tumor. They have been reported in thoracolumbar spine and lower cervical spine ${ }^{10}$. Our case, a female, has a tumor located in the axis with extension into $\mathrm{C} 3$ and then caudally to $\mathrm{C} 4$ as the disease progressed. We present a technical innovation in spinal stabilization and compared our result with previously reported cases.

KEY WORDS: Chondrosarcoma. Spine tumor. Cervical spine. Instability

\section{Caso clínico}

Mujer de 61 años de edad con antecedentes patológicos de hipertensión arterial controlada farmacológicamente y dos episodios de sinusitis maxilar derecha tratados médicamente cuatro y dos años previos al problema actual. Sin alergias conocidas y sin intervenciones quirúrgicas

Recibido: 17-02-04. Aceptado: 26-03-04 previas. Presenta en abril 1999 tumoración subcutánea laterocervical derecha a nivel del ángulo de la mandíbula, que inicialmente se relaciona con un cuadro de otitis aguda ocurrido dos meses antes. Desde la aparición del bultoma, refiere dolor cervical moderado que se acentúa con movimientos de rotación.

En junio del mismo año refiere disestesias en hombro y extremidad superior derecha asociados a dolor cervical, así como neuralgia de $\mathrm{C} 2$ ipsilateral.

Exploración: se aprecia una tumoración de características gomosas bien definida en el ángulo mandibular derecho adherida a planos profundos y moderadamente dolorosa a la palpación. No existe alteración de vías largas y los pares craneales están conservados bilateralmente.

Investigaciones: una analítica completa no revela ninguna anormalidad. Se procede a la práctica de una RMN cervical apreciándose una lesión ocupante de espacio, que ocupa el foramen de conjunción derecho de C2-C3 con ampliación del mismo y erosión de la masa lateral del axis, así como de la articulación interapofisaria posterior derecha. Se observa ocupación del receso anterolateral del canal raquídeo pero sin distorsión del cordón medular. Anteriormente distorsiona, desplaza y colapsa la arteria vertebral derecha y contacta con la bifurcación carotídea y la vena yugular interna sin afectarlas. También distorsiona la musculatura laterocervical, pero sin infiltrarla (Fig. 1). A continuación se realiza un estudio TAC en el que se aprecia, más claramente, una erosión de la masa lateral de C2 (Fig. 2). Por último, una angiografía de troncos supraórticos demuestra la oclusión del flujo vertebral derecho.

Manejo: en abril 2000 se procede conjuntamente con el servicio de ORL, a practicar una resección subtotal de dicha tumoración por vía cervical lateral, con el fin de obtener diagnóstico histológico y reducir la masa tumoral. E1 procedimiento transcurre sin incidentes, dándose el alta cinco días más tarde. La paciente permanece asintomática.

Una vez obtenido el diagnóstico de condrosarcoma grado I bien diferenciado, se reingresa a la paciente, un

Abreviaturas. ORL: Otorrinilaringología 


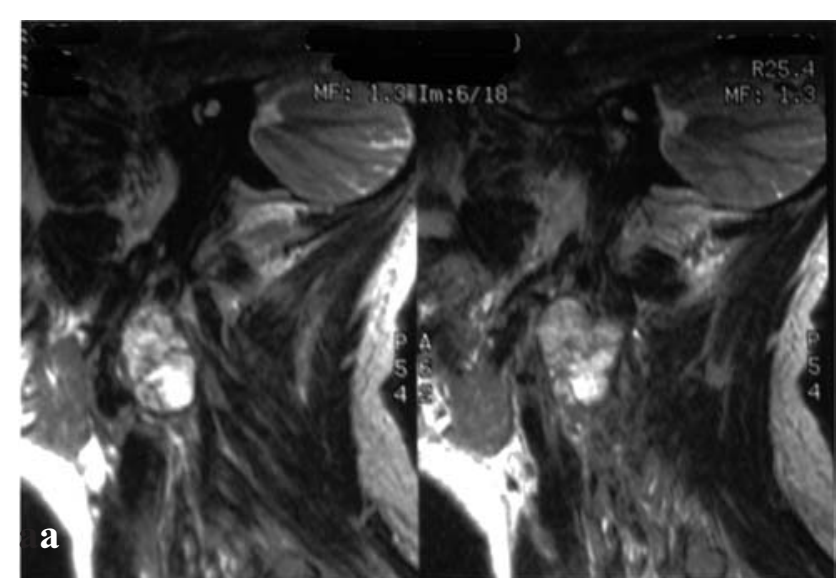

Figura 1. RMN cervical apreciándose masa de tejido en íntimo contacto con paquete vascular y esterno cleidomastoideo (a). Se observa el cordón medular libre de compresión (b).

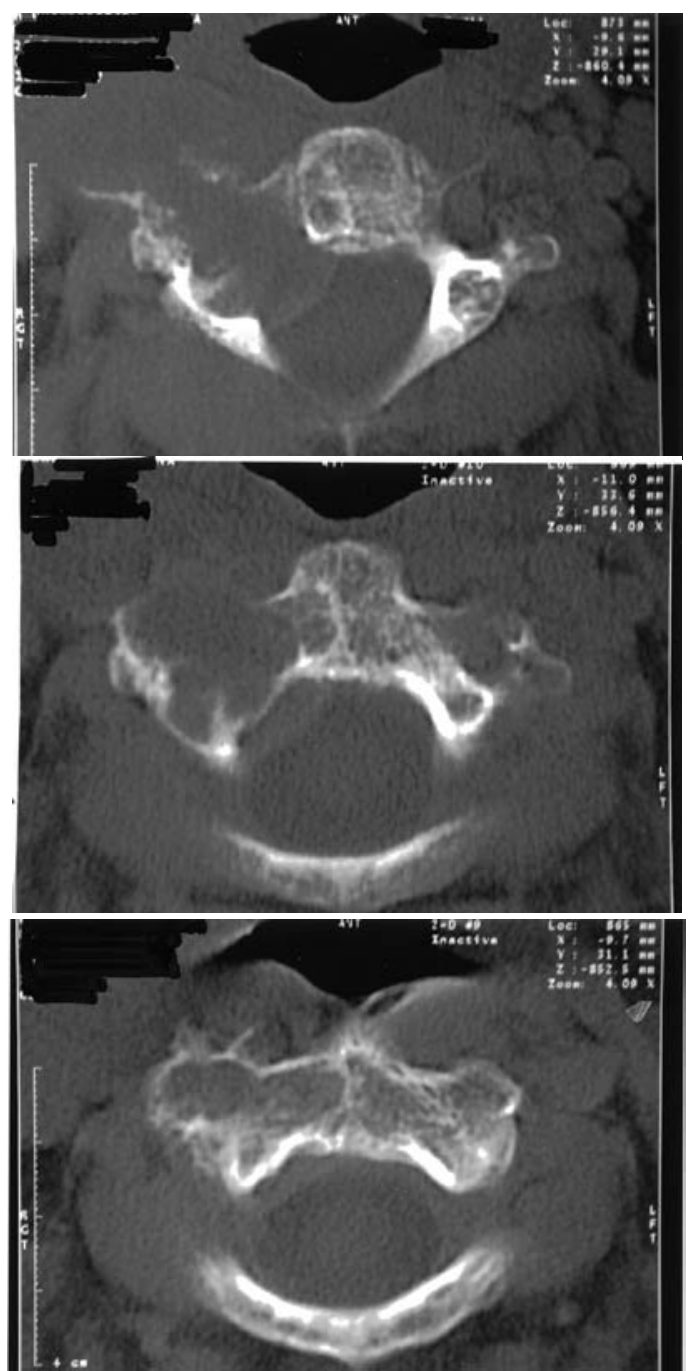

Figura 2. TAC cervical, erosión e insuflación de la masa lateral de C2. Masa de tejido blando que no compromete el cordón medular pero que agranda el foramen de conjunción y oblitera la arteria vertebral derecha.
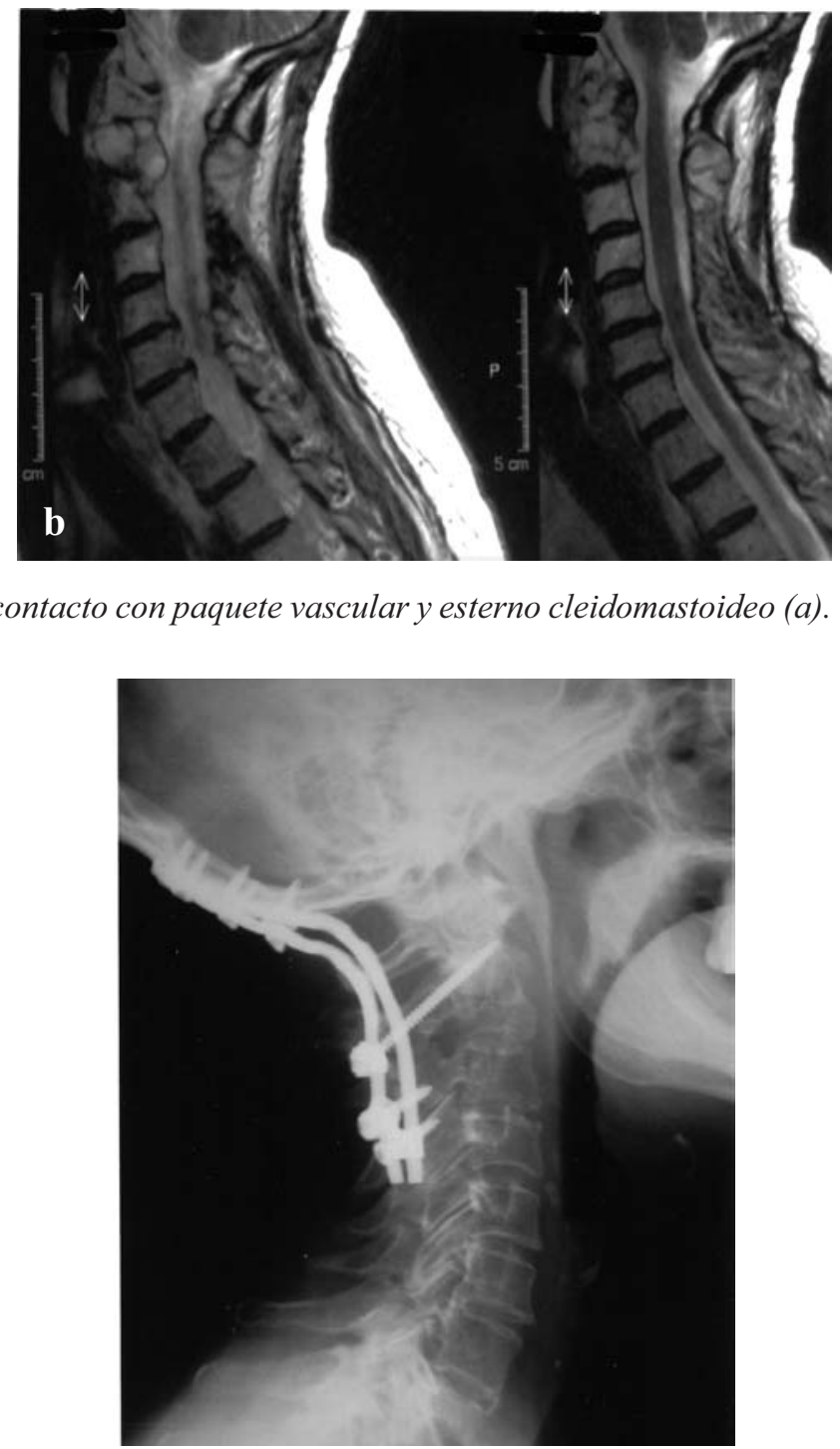

Figura 3. Fijación occipito-cervical, con tornillo transpedicular de C2 en el lado izquierdo. Masa lateral de C2 derecha está destruida por el tumor así como parte de C3 ipsilateral condicionando el atornillado a masa lateral.

mes más tarde, para intentar completar la resección por vía posterior, así como conferir estabilidad a la columna cervical alta por medio de una fijación instrumentada occipito-cervical. Durante el procedimiento que transcurre satisfactoriamente, se aprecia una tumoración que erosiona, insufla e invade los macizos articulares de $\mathrm{C} 2$ y C3 derechas así como sus hemiláminas respectivas. También se observa una invasión del hemicuerpo derecho del axis. La arteria vertebral se encuentra trombosada. Las raíces de $\mathrm{C} 2$ y $\mathrm{C} 3$ derechas quedan indemnes y el saco dural libre. La instrumentación realizada consistió en el atornillado transpedicular de C2 izquierdo electivamente, según la técnica de $\mathrm{Goel}^{4}$, con extensión craneal anclando 
tres tornillos en la escama occipital bilateralmente, y con extensión caudal con atornillado en masa lateral de C3 izquierda y masa lateral derecha de C4 (Fig. 3). Se consigue una exéresis subtotal. El curso postoperatorio es totalmente satisfactorio, mostrando la radiografía de control postoperatoria una correcta fijación. Se procede al alta domiciliaria nueve días más tarde, remitiendo al paciente al servicio de radioterapia donde se le administra un curso de 50 Gy como tratamiento coadyuvante.

Evolución: en abril 2002 se constata la presencia de una adenopatía laterocervical derecha. Un nuevo estudio RMN constata la recidiva tumoral. Se practica exéresis de la adenopatía y tras análisis histológico confirma la presencia de recidiva, procediéndose a braquiterapia con $40 \mathrm{~Gy}$.

En marzo 2003 se comprueba nuevo aumento tumoral; se decide nueva intervención quirúrgica, después de demostrar la no existencia de enfermedad a distancia. En esta ocasión se practica abordaje transcervical lateral suprahioideo con resección subtotal. También se practica traqueostomía electiva. Ambos procedimientos transcurren sin incidencias.

Desafortunadamente, tres meses más tarde se vuelve a confirmar nuevo crecimiento tumoral coincidiendo con dolor en hemifacies izquierda irradiado a extremidad superior izquierda decidiéndose tratamiento sintomático. En diciembre 2003 coincidiendo con la presencia de dificultad motora en ambas extremidades superiores se confirma radiológicamente nuevo crecimiento tumoral. En esta ocasión se desestima nueva intervención quirúrgica y se decide la administración de quimioterapia siguiendo un esquema de adriamicina + ifosfamida. Tras la administración de dos ciclos se abandona el tratamiento por pobre respuesta y alta toxicidad.

\section{Discusión}

El condrosarcoma es un tumor maligno de células productoras de cartílago. Se puede clasificar de varias formas. Dependiendo de su grado histológico; si es primario (de novo) o secundario (asociado a una lesión preexistente) y si es central o periférico -con respecto a su localización en el canal medular óseo o en la superficie de este ${ }^{7}$-. De estas clasificaciones, la que ayuda más a establecer la prognosis es la primera. Para mayor complicación, la clasificación histológica se puede dividir en subtipos como: condroarcoma de células claras descrito por Unni en $1976^{9}$, mesenquimal, de base de cráneo y de partes blandas.

La clasificación en la que se basa nuestro caso es la que se divide en grado bajo (grado 1), medio (grado 2) y alto (grado 3). Esta última es la que tiene mayor posibilidad de metastatizar. El grado está basado en las apariencias histológicas del tumor. Aquellos que se asemejan más a cartílago normal se denominan de grado 1 y aquellos que recuerdan a cartílago anormal reciben el calificativo de grado 3. Existe aun otro tipo de condrosarcoma con mayor posibilidad de metástasis que el de grado 3, el llamado condrosarcoma desdiferenciado, siendo este último extremadamente raro. Probablemente se origina de lesiones benignas de cartílago o bien de condrosarcomas de bajo grado. El examen histológico revela la presencia de células malignas en huso que no se asemejan a células de origen cartilaginoso; existen áreas adyacentes de condrocitos neoplásicos rodeados de una matriz mixta de cartílago-hialina.

Demográficamente, existe una mínima preponderancia del sexo masculino sobre el femenino con respecto a todos los condrosarcomas. Con respecto a la edad de presentación, la mayoría de los casos se encuentran entre la cuarta y séptima décadas de la vida, aunque no es raro encontrarlos en pacientes más jóvenes. Nuestro caso sólo cumple uno de los dos datos antes citados.

El lugar típico en el que se presenta el condrosarcoma es la pelvis $(24 \%)$ y la parte proximal del fémur $(16 \%)$. Se han descrito otras localizaciones que, a pesar de no ser $\tan$ frecuentes, no se pueden considerar raras, como por ejemplo las costillas o la escápula. A nivel espinal, ya hemos comentado que se trata de presentaciones inusuales, entre $2-10 \%^{2}$, ya descritas. Sobre todo a nivel torácico, con más frecuencia que lumbar. A nivel cervical existen casos puntuales publicados; estos están confinados a nivel cervical medio-bajo $\mathrm{C} 4$ y $\mathrm{C} 7$ e incluso a nivel intraespinal ${ }^{10}$. En nuestro caso, se encontraba inicialmente en C2-3 con extensión a $\mathrm{C} 4$ durante la evolución de la enfermedad.

Clínicamente, los condrosarcomas se manifiestan con dolor, probablemente por distensión y más tarde destrucción del periostio, así como deformidad. Tanto uno como otro pueden ser invalidantes e interferir con la calidad del sueño. Nosotros podemos confirmar esta observación y añadir que, en el caso de localización espinal, también pueden darse signos y síntomas relacionados con inestabilidad funcional, así como compresión de estructuras vasculares y nerviosas, como se puso de manifiesto en la evolución de la enfermedad de nuestro paciente.

Radiológicamente, el estudio se inicia con estudios simples de la parte afectada, que en nuestro caso se ampliaron a estudios funcionales, con el fin de valorar la estabilidad cervical. A continuación, y por razones de infraestructura del servicio de radiodiagnóstico, se procedió a la práctica de un TAC cervical que nos permitió valorar con más deta1le la erosión ósea de C2 (Fig. 2), así como la presencia de una masa de tejido blando. En el caso de sospecha de compromiso medular, como fue nuestro caso, se procede a la realización de una RMN (Fig. 1), que completamos con estudios de angiografía, con el fin de corroborar la afectación vertebral sospechada en el TAC y RMN.

En un principio, por tratarse de un condrosarcoma 
de bajo grado y, por lo tanto, con pocas posibilidades de metástasis, no se procedió a estudios de extensión. Estos se llevaron a cabo, previo al inicio de la quimioterapia, por aumentar las posibilidades de mayor malignización. Se realizó un body TAC incluyendo tórax, abdomen y pelvis. De estos, los depósitos metastásicos tienen predilección por el primero ${ }^{7}$. Dichos estudios no revelaron enfermedad a distancia. Es interesante mencionar que, dentro de los diagnósticos diferenciales, se debe incluir los casos de mieloma y depósitos metastásicos de carcinomas provenientes de pulmón, mama, tiroides, riñón y próstata.

El abordaje terapéutico de estos casos es complejo, incluyendo cirugía resectiva-estabilizadora, radioterapia así como braquiterapia y quimioterapia.

En el caso de condrosarcomas de localización extraespinal el tratamiento de elección, y por ende con mejores resultados, es la resección total con amplios márgenes. De hecho, el condrosarcoma en general no infiltra tejidos vecinos permitiendo un buen plano de clivaje. En ocasiones, se opta por una biopsia inicial para tener confirmación histológica, teniendo especial precaución con la posibilidad de diseminación local en el trayecto biopsiado.

En nuestro caso, y de manera electiva, se decidió realizar una resección parcial por vía anterior, comprobándose que a pesar de estar en íntimo contacto con el músculo esternocleidomastoideo y el paquete vascular yúgulo-carotídeo estos no se hallaban infiltrados. Se opto por dejar el abordaje posterior para una segunda ocasión.

El segundo tiempo quirúrgico planteaba problemas de alto riesgo. El hecho de conseguir una resección total implicaba desestabilizar el segmento occipitocervical y poner en riesgo la circulación de la única arteria vertebral funcionante así como el potencial daño neurológico. Se procedió a la fijación occipito-cervical incluyendo masas laterales de $\mathrm{C} 4$ en el lado derecho y $\mathrm{C} 3$ en el izquierdo. A nivel del axis se introdujo un tornillo transpedicular en el lado izquierdo dejando el lado contralateral sin atornillado. Posteriormente se vació el tumor que destruía la articulación C2-3. Al final del procedimiento se obtuvo una fijación estable y sólida. Se procedió con radioterapia por existir evidencia de enfermedad residual. Harwood et $\mathrm{al}^{5}$ sugieren que la radioterapia a alta dosis (60 Gy) enlentece el crecimiento del condrosarcoma y debe reservarse para casos en los que la resección quirúrgica ha sido insuficiente o imposible.

Hay otros autores que sugieren "proton beam" como alternativa para condrosarcomas irresecables de columna ${ }^{8}$.

Durante el seguimiento del paciente se constata clínica y radiológicamente progresión de la enfermedad. En esta ocasión se optó por un curso de braquiterapia. Desafortunadamente, la enfermedad siguió progresando, procediéndose a un nuevo curetage de la lesión que permitió un nuevo enlentecimiento de la enfermedad durante nueve meses.
Después de los cuales se intentó sin éxito el tratamiento con quimioterapia. Esta se reserva principalmente en casos donde existe enfermedad metastásica desde el inicio ${ }^{5}$, pero en nuestro caso se optó por esta modalidad por ser el último recurso disponible.

La supervivencia del paciente coincide con los datos existentes en la literatura ${ }^{2,10}$. Nuestro caso supera los 60 meses de supervivencia. Cabe destacar que el índice de supervivencia mejora si la resección se realiza en bloque, si es posible, comparado con técnicas de curetage.

\section{Conclusiones}

El condrosarcoma es generalmente un tumor de bajo grado y crecimiento lento. A pesar de los avances en diagnóstico por neuroimagen y la mejora de las técnicas quirúrgicas de instrumentación espinal, así como la radio y quimioterapia adjuvantes, el tratamiento de estas lesiones continúa siendo insatisfactorio, debido al gran índice de recurrencia.

Varios factores influyen en la pobre prognosis de pacientes portadores de condrosarcomas, como su edad al comienzo de los síntomas, el patrón tumoral con sus anormalidades citogenéticas y la extensión de la resección quirúrgica.

El tratamiento de elección siempre que sea posible es la resección en bloque, dejando la radioterapia para los casos en que la resección sea parcial o el tumor continúe creciendo. La quimioterapia se reserva para aquellos casos en los que haya enfermedad metastásica.

\section{Bibliografía}

1. Blaylock, R.L., Kempe, L.G.: Chondrosarcoma of the cervical spine. Case report. J. Neurosurg. 1976; 44: 500-503.

2. Boriani, S., De Lure, E., Bandiera, A., et al: Chondrosarcoma of the mobile spine. Report on 22 cases. Spinei 2000; 25: 804-812.

3. Bourgoin, P.M., Tampieri, D., Robitafle, Y., et al: Lowgrade miyxoid chondrosarcoma of the base of the skull: CT, MR and histopathology. J Comput Assist Tomogr. 1992; 16: 268-273.

4. Goel, A., Lahen, V P.:late and screw fixation for adantoaxial subluxation. Acta Neurochir (Wien). 1994; 129: 4753.'

5. Harwood, A.R., Krajbich, J.I. Fornasier, V.L.: Radiotherapy of chondrosarcoma of bone. Cancer. 1980; 45: 27692777.

6. Ranjan, A., Chacko, G., Joseph, T., et al: Intraespinal mcsenchymal chondrosarcoma. J. Neurosurg. 1994; 80: 928-930.

7. Springfield, D.S., Gebhart, M.C., Mcguire, M.H.: Chondrosarcoma a review. J. Bone J. Surg. 1996; 78:141-149.

8. Suit, H.D., Goitin, M., Munzenreider, J., et al.: Definitive radiation therapy for chordoma and chondrosarcooma of 
the base of the skull and cervical spine. J. Neurosurg. 1982; 57: $377-85$.

9. Unni, K.K., Dahlin, D.C., Beabout, J.W., et al: Chondrosarcoma: clear cell variant. A report of sixteen cases. J. Bone J. Surg. 1976; 58-A: 676-683.

10. York, J.E., Berk, R.H., Fuller, G.N., et al: Chondrosarcoma of the spine: 1954 to 1997. J Neurosurg (Spine 1). 1999; 90: 73-78.
Domínguez, C.J.; Martín-Ferrer, S.; Rimbau, J.; Joly, C.: Condrosarcoma cervical alto. Neurocirugía 2005; 16 : 261-265.

Correspondencia postal: Carlos J. Domínguez. Servicio de Neurocirugía. Hospital Universitari de Girona. Avd. de Franja s/n. 17007 Girona

Comentario al trabajo: Condrosarcoma cervical alto de C. J. Dominguez y cols.

El Dr. C.J. Domínguez y colaboradores describen el caso de una paciente portadora de un condrosarcoma grado 1 a nivel C2-C3 y seguida durante cuatro años.

Se le efectúa resección parcial de la lesión en tres momentos diferentes de la evolución.

Durante el segundo procedimiento utilizan una fijación occipito-cervical tipo Goel con lo que consiguen una buena estabilidad mecánica de la columna.

Esta lesión de baja malignidad no se mostró sensible a la radio o quimioterapia; por lo que nos hace suponer la justificación de un tratamiento quirúrgico lo más agresivo posible buscando la resección completa con márgenes y en bloque si es posible.

\section{J.J. Acebes}

Barcelona 